\title{
Conjugation fidelity and reflectivity in photorefractive double phase-conjugate mirrors
}

\author{
Sergei Orlov, Mordechai Segev, and Amnon Yariv \\ California Institute of Technology, 128-95, Pasadena, California 91125 \\ George C. Valley \\ Hughes Research Laboratories, 3011 Malibu Canyon Road, Malibu, California 90265 \\ Received December 20, 1993

\begin{abstract}
We present an experimental study of the behavior of photorefractive double phase-conjugate mirrors that illustrates recent theoretical predictions. We observe a sharp fidelity threshold that significantly depends on the specific feature size in the input beams. Furthermore, we find that if the two input beams have unequal intensities the conjugation process is asymmetric and the steady-state fidelity is better on the side of the crystal on which the more-intense beam enters.
\end{abstract}

The double phase-conjugate mirror ${ }^{1}$ (DPCM) is a unique photorefractive (PR) device in which two mutually incoherent input beams become phase conjugates of each other (i.e., the beams exchange slowly varying amplitude and phase profiles). As the DPCM develops, the input beams fan and at the same time scatter from each other's fanning gratings. This process is completely phase matched only if the beams are phase conjugates of each other throughout the entire volume. ${ }^{2}$ Therefore a selection mechanism in which common gratings are enhanced and nonoverlapping gratings are suppressed governs the evolution. Eventually, if the gain (average coupling coefficient times interaction length) is large enough, the interaction stabilizes in a double phase-conjugation form.

In a recent Letter ${ }^{3}$ we presented a theoretical model for the evolution of the DPCM that starts from two input beams and randomly scattered noise. We predicted that there exists a fidelity threshold for the double-conjugation process. More recent calculations suggest that the threshold in the gain depends on the feature size (or the resolution) in the input beams. ${ }^{4}$ In particular, we find that in a complicated image of a varying resolution the large features possess a lower gain threshold and appear earlier in time, whereas the small features (high resolution) suffer from a higher threshold. In this respect the finest feature that may be conjugated in a specific device is limited by the available gain; i.e., very high-resolution images may not be conjugated in a specific device. On the other hand, the phaseconjugate reflectivity is a smoothly increasing function of the gain. In later calculations we have also found that as we vary the input beams' intensity ratio the threshold value increases (as expected ${ }^{1}$ ), but the conjugation process becomes asymmetric; the steadystate fidelity is improved for the stronger input beam and reduced for the weaker one. Note that Mamaev and Shkunov ${ }^{5}$ observed an increase in the threshold for the reflectivity after introduction of fine-scale features (speckles) in the input beams. This observation was attributed ${ }^{6}$ to the nonlinear intensity dependence of the PR gratings.

In this Letter we present experimental evidence that supports our theoretical prediction of a threshold in the conjugation fidelity of the DPCM. In addition, we observe that the fidelity threshold depends on the feature size in the conjugated images. The phaseconjugate reflectivity, however, is a smooth function of the gain. We have also modified the input beams' intensity ratio until one output is well phase conjugated while the other output is masked by the noise. These results are useful for an understanding of the limitations of device applications, such as in image processors, ${ }^{1,7}$ interconnects, ${ }^{8}$ interferometers, ${ }^{9}$ and coupled-laser devices. ${ }^{10}$

Our experimental setup, which is nearly identical to the standard DPCM configuration, ${ }^{1}$ consists of an Ar-ion laser, a beam splitter, and two transparencies followed by beam splitters and CCD cameras located on opposite sides of a $\mathrm{BaTiO}_{3}$ crystal. The multimode 488-nm Ar-ion laser has a 3-cm coherence length and is polarized in the plane of the optical system (extraordinary polarization). Each beam acquires an image by propagating through a U.S. Air Force resolution chart. After passing through an additional beam splitter and an imaging system, both beams recombine at the $\mathrm{BaTiO}_{3}$ crystal. Care is taken to ensure that the beams are mutually incoherent at the crystal plane (the optical path difference between the arms is much larger than the coherence length). To facilitate comparison with our modeling, we place the crystal between the image and the focal planes of the U.S. Air Force resolution chart so that the transverse intensity distribution in the crystal is nearly uniform. To vary the PR coupling coefficient, we uniformly illuminate the crystal from above with a third, erasure, beam from the Ar-ion laser that is incoherent with respect to the DPCM beams and has ordinary polarization to eliminate fanning of the erase beam. Changing the 
intensity of the erase beam varies the visibility of the DPCM interference patterns and hence the modulation depth of the index gratings or the PR coupling coefficient throughout the crystal. We measure the conjugation fidelity and reflectivity of the DPCM, using the backpropagating reflections from the beam splitters in both arms. These reflections are magnified and captured by a CCD camera. When the fidelity of the conjugated images is measured, we use a scanning variable slit across the magnified image plane and measure the transmitted power in different locations. In this manner we are able to compare the conjugation fidelity of the bars of a specific resolution with the original contrast of the resolution chart. The best conjugate image obtained from our DPCM is shown in Fig. 1(a); Fig. 1(b) shows the magnified central portion of the chart. The best resolution in the phase-conjugate image is $40 \mu \mathrm{m}$ (across the entire image), limited only by the apertures of our optical components, and the maximum observed phase-conjugate reflectivity is $35 \%$.

An important issue in the operation of the DPCM is the orientation of the structure within the input images with respect to the main plane of interaction (defined by the largest average projection of the gratings and the polarization of the beams). When all the bars in an image are perpendicular to the plane of interaction, Bragg degeneracy results in the formation of conical rings (in the general direction of the bars). This effect cannot be accounted for by our two-dimensional theoretical model ${ }^{3}$ and requires a full three-dimensional analysis. We overcome these problems simply by a $45^{\circ}$ rotation of the images, which provides grating distributions that are neither parallel or perpendicular to the main plane of interaction. In this respect, our current results never suffer from Bragg degeneracy, and we obtain equal fidelities for bars in both directions. We note another method for suppression of Bragg degeneracy that uses speckles borne on the input beams. ${ }^{11}$

In the first experiment we measure the conjugation fidelity and the conjugation reflectivity of a specific group of elements in the image (resolution chart). We concentrated on element \#1, group \#2, which consists of three bars at the density of 4.00 line pairs $/ \mathrm{mm}$ (bar width, $125 \mu \mathrm{m}$ ) in the original image plane. Because in the original image all the bright bars have equal intensities, it is essential to account for the relative intensities in the reconstruction plane. Hence we use a measure for the conjugation fidelity, given by

$$
F=\frac{0.5\left(v_{1}+v_{3}\right)-v_{2}}{\max \left(v_{1}, v_{3}\right)+v_{2}},
$$

where $v_{1}, v_{2}$, and $v_{3}$ are the optical intensities measured in a sequence of bars that are brightdark-bright in the original image. For example, if the conjugate image is bright-dark-bright, $F$ would be 1; if the conjugate image is bright-dark-dark, $F=1 / 2$; if the conjugate image is reversed (dark-bright-dark), $F=-1$; and if the conjugate image is bright-bright-bright, $F=0$. We point out that $F$ does not equal the conjugation fidelity of Ref. 3, although they have similar qualitative behavior (there we defined fidelity in the Fourier plane, where phases are crucial; here we compare intensity distribution of the original and conjugated images; the slit provided averaging along the bright-dark bars).

The normalized conjugation reflectivity is defined as the intensity integrated over the entire region of the image, $v_{1}+v_{2}+v_{3}$, divided by its maximal obtainable value (at the highest available gain). We measure the fidelity and the reflectivity as function of the power of the erasure beam $I_{e}$. Since the $P R$ perturbation in the refractive index is proportional to the visibility of the corresponding interference fringes, the coupling coefficient $\gamma$ may be rewritten ${ }^{12}$ to incorporate the reduction in the visibility resulting from $I_{e}$ as

$$
\gamma\left(I_{e}\right)=\frac{\gamma_{0}}{1+\left(I_{e} / I_{0}\right)},
$$

where $I_{0}=I_{1}+I_{2}, I_{1}$ and $I_{2}$ are the intensities of the interacting beams and $\gamma_{0}$ is the coupling constant for $I_{e}=0$. The PR gain is defined as coupling coefficient $\gamma$ times the interaction length $L$ (in our experiment $L=7 \mathrm{~mm}$ ). The results shown in Fig. 2 reveal a sharp fidelity threshold at $I_{e}=0.55$ (in arbitrary units). From the optical power of the input beams and the beams' cross sections, we estimate the average $I_{0}$ to be roughly $0.32 \pm 0.05$ (in arbitrary units of the scale of Fig. 2). We have measured the average coupling coefficient in this configuration by a standard small-signal amplification experiment. This measurement provides a $\gamma$ value of $8 \mathrm{~cm}^{-1}$, which gives roughly a gain-threshold value of 2.06 (because beam fanning drives down the value of $\gamma$ for these measurements, we suspect that the true value
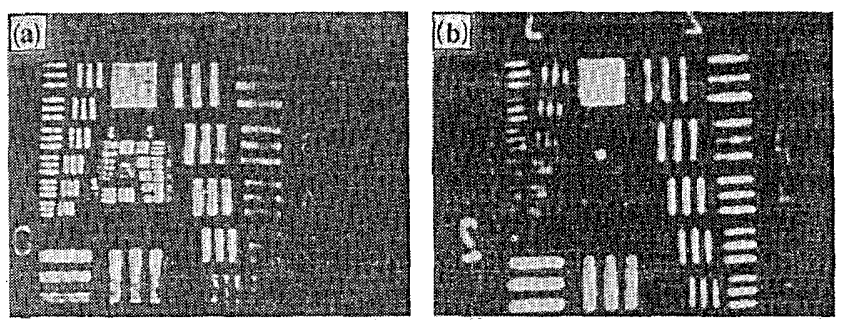

Fig. 1. Phase-conjugate images of (a) the entire resolution chart and (b) the inner frame of (a).

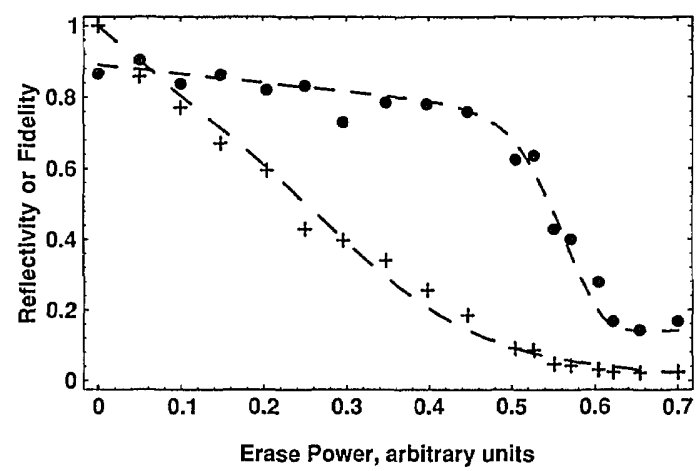

Fig. 2. Phase-conjugation fidelity (circles) and reflectivity (crosses) as a function the power of the erasure beam. Note the fidelity threshold at erase power of 0.55 a.u. The curves are only a guide to the eye. 


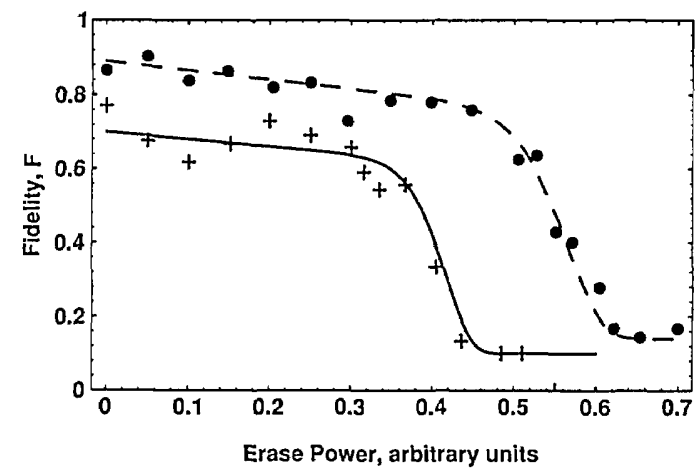

Fig. 3. Phase-conjugation fidelity as a function the power of the erasure beam for high (crosses) and low (circles) resolutions. The curves are only a guide to the eye.
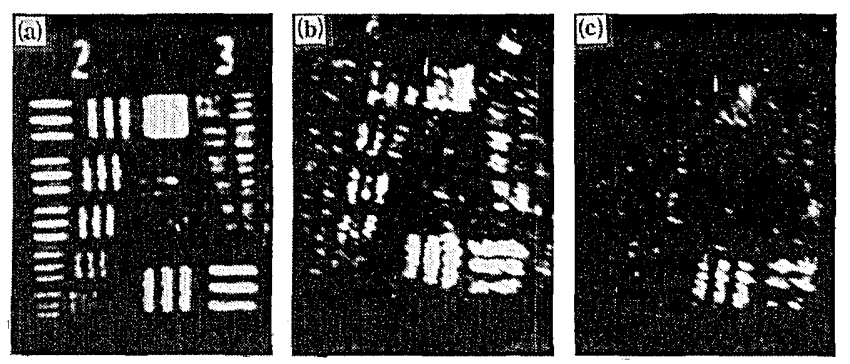

Fig. 4. Phase-conjugate images of (a) maximal, (b) high, and (c) low gain for an input image that contains both resolutions. Note that most of (c) is below threshold.


Fig. 5. Phase-conjugate images for unbalanced input beams of intensity ratio 1:5: (a) the beam that leaves the face on which the beam of intensity 1 enters, (b) the beam that leaves the face on which the beam of intensity 5 enters.

is somewhat higher). The conjugation reflectivity, compared with the fidelity, exhibits a smooth behavior. We notice that the reflectivity does saturate at high gains but does not display a threshold behavior as does the fidelity. Intuitively, it is clear that conjugation reflectivity cannot provide accurate data for characterization of the DPCM device, simply because part of this reflection is amplified noise (fanning), which may be distinguished from the reflectivity of the desired image only by fidelity measurements.

In the second experiment we measure the conjugation fidelity as a function of the resolution (feature size) within the conjugated image. Figure 3 shows the conjugation fidelity for two cases: high resolution, 6.36 line pairs/mm (bar width, $78.74 \mu \mathrm{m}$; element \#5, group \#2; solid curve) and low resolution, 4.00 line pairs $/ \mathrm{mm}$ (bar width, $125 \mu \mathrm{m}$; element \#1, group \#2; dashed curve). Note that we use the same arbitrary units for $I_{e}$ in both Figs. 2 and 3. Figure 3 clearly shows that the gain-threshold value significantly differs in both cases: the lower resolution has a lower threshold value. To emphasize this we show Fig. 4 with both resolutions conjugated [Fig. 4(a) maximal gain, $I_{e}=0$; Fig. 4(b) relatively high gain, small $I_{e}$ ] and only the low resolution conjugated [Fig. 4(c) low gain, large $I_{e}$ ].

In the third experiment we keep the gain fixed (at $I_{e}=0$ ) but modify the intensity ratio of the input beams from unity to 1:5. In this case we find asymmetry in the conjugation process. Specifically, the stronger beam bleaches the fanning (amplified noise) gratings of the weak beam and permits relatively high-conjugation quality on its input side (i.e., high fidelity is obtained for the image originally borne on the strong beam and transferred to the weak beam). The opposite process, however, is inefficient because of the unbalanced process, and the weak beam is not able to eliminate the appearance of fanning gratings, which result in a conjugate image embedded in fanning. This is shown in Fig. 5. Note the difference between this result on fidelity and the known result ${ }^{1}$ (which is present here as well) of an increase in conjugation reflectivity threshold as a result of unbalanced beams.

In conclusion, we present experimental results that substantiate our earlier theoretical prediction of a sharp threshold in the conjugation fidelity of the double phase-conjugate mirror measured as a function of coupling coefficient.

This study was supported by the Advanced Research Projects Agency, the U.S. Army Research Office, and the U.S. Air Force Office of Scientific Research.

\section{References}

1. S. Weiss, S. Sternklar, and B. Fischer, Opt. Lett. 12, 114 (1987) (see Fig. 1); Appl. Phys. Lett. 50, 483 (1987) (see Fig. 1); Opt. Eng. 26, 423 (1987) (see Fig. 3).

2. M. Segev and A. Yariv, Opt. Lett. 16, 1938 (1991).

3. M. Segev, D. Engin, A. Yariv, and G. C. Valley, Opt. Lett. 18, 1828 (1993).

4. The note added in proof at the end of Ref. 3 was incorrectly printed. It should read "the threshold value... decreases with increasing feature size ....".

5. A. V. Mamaev and V. V. Shkunov, Kvantovaya Elektron. (Moscow) 19, 1111 (1992) [Sov. J. Quantum Electron. 22, 1036 (1992)].

6. O. L. Lyubomudrov and V. V. Shkunov, Kvantovaya Elektron. (Moscow) 19, 1102 (1992) [Sov. J. Quantum Electron. 22, 1027 (1992)].

7. R. J. Anderson, E. J. Sharp, G. L. Wood, W. W. Clark, Q. Vuong, G. J. Salamo, and R. R. Neurgaonkar, Opt. Lett. 18, 986 (1993).

8. S. Weiss, M. Segev, S. Sternklar, and B. Fischer, Appl. Opt. 27, 3422 (1988).

9. S. Sternklar, S. Weiss, M. Segev, and B. Fischer, Appl. Opt. 25, 4518 (1986).

10. S. Sternklar, S. Weiss, M. Segev, and B. Fischer, Opt. Lett. 11, 528 (1986); M. Segev, S. Weiss, and B. Fischer, Appl. Phys. Lett. 50, 1397 (1987).

11. A. V. Mamaev and V. V. Shkunov, Kvantovaya Elektron. (Moscow) 17, 389 (1990) [Sov. J. Quantum Electron. 20, 323 (1990)].

12. S. K. Kwong, M. Cronin-Golomb, and A. Yariv, Appl. Phys. Lett. 45, 1016 (1984). 